\title{
Modulation of G6PD affects bladder cancer via ROS accumulation and the AKT pathway in vitro
}

\author{
XIAOYI CHEN ${ }^{1 *}$, ZHIJIE XU $^{1 *}$, ZHIJIAN ZHU $^{1}$, ANQI CHEN ${ }^{2}$, \\ GUANGHOU FU $^{1}$, YIMIN WANG ${ }^{1}$, HAO PAN ${ }^{1}$ and BAIYE JIN ${ }^{1}$ \\ ${ }^{1}$ Department of Urology, The First Affiliated Hospital, School of Medicine, Zhejiang University, \\ Hangzhou, Zhejiang 310009; ${ }^{2}$ Department of Materials Science and Engineering, \\ College of Engineering, Peking University, Beijing 100871, P.R. China
}

Received March 13, 2018; Accepted July 9, 2018

DOI: 10.3892/ijo.2018.4501

\begin{abstract}
Glucose-6-phosphate dehydrogenase (G6PD) is a rate-limiting enzyme of the pentose phosphate pathway. Multiple studies have previously revealed that elevated G6PD levels promote cancer progression in numerous tumor types; however, the underlying mechanism remains unclear. In the present study, it was demonstrated that high G6PD expression is a poor prognostic factor in bladder cancer, and the levels of G6PD expression increase with increasing tumor stage. Patients with bladder cancer with high G6PD expression had worse survival rates compared with those with lower G6PD expression in resected tumors. In vitro experiments revealed that knockdown of G6PD suppressed cell viability and growth in Cell Counting Kit- 8 and colony formation assays, and increased apoptosis in bladder cancer cell lines compared with normal cells. Further experiments indicated that the weakening of the survival ability in G6PD-knockdown bladder cancer cells may be explained by intracellular reactive oxygen species accumulation and protein kinase B pathway suppression. Furthermore, it was additionally revealed that 6-aminonicotinamide (6-AN), a competitive G6PD inhibitor, may be a potential therapy for bladder cancer, particularly in cases with high G6PD expression, and that the combination of cisplatin and 6-AN may optimize the clinical dose or minimize the side effects of cisplatin.
\end{abstract}

\section{Introduction}

Bladder cancer is the most common urinary malignancy in China, with an annual incidence rate and estimated mortality

Correspondence to: Dr Baiye Jin, Department of Urology, The First Affiliated Hospital, School of Medicine, Zhejiang University, 79 Qingchun Road, Hangzhou, Zhejiang 310009, P.R. China

E-mail: 1189006@zju.edu.cn

*Contributed equally

Key words: glucose-6-phosphate dehydrogenase, bladder cancer, reactive oxygen species accumulation, protein kinase B pathway, 6-aminonicotinamide, cisplatin rate of $80.5 / 100,000$ and $32.9 / 100,000$, respectively (1). Despite recent improvements in treatment strategies, the overall survival rates of patients with an advanced stage of the disease remains poor (2). Tumor progression and metastasis are the main causes of bladder cancer-associated mortality, yet the exact mechanisms underlying these processes have not been fully elucidated. Hence, it is imperative to determine the key mechanisms implicated in bladder cancer development and progression, in order to identify potential therapeutic targets to improve patient prognosis.

The pentose phosphate pathway (PPP), one of the alternative routes for glucose metabolism, provides products for biosynthesis and antioxidant defense in cells (3). PPP has been attracting increased attention due to its ability to facilitate tumor progression or chemotherapy resistance by satisfying the considerable biosynthetic demands of rapidly growing cancer cells, in addition to their resistance and survival under stress conditions (3). Glucose-6-phosphate dehydrogenase (G6PD) is a rate-limiting enzyme of the PPP, and it is well known to promote intracellular anabolic reactions and redox homeostasis (4). Recently, multiple studies have demonstrated that elevated G6PD levels promote cancer progression in numerous tumor types, including melanoma, leukemia and colon cancer (5-10). Considering the function of G6PD in the critical processes of cancer cells, it is imperative to identify the mechanisms underlying the function of G6PD in bladder cancer in order to develop potent and selective G6PD inhibitors (10).

The aim of the present study was to investigate whether the high expression of G6PD in bladder cancer is associated with tumor aggressiveness and poor clinical prognosis and to determine whether targeting G6PD may be of value as a therapeutic option for bladder cancer, particularly in advanced cases.

\section{Materials and methods}

Online database. Information on G6PD mRNA expression in bladder cancer and normal tissues were acquired from the Oncomine database (https://www.oncomine.org) using the following searching terms: G6PD and bladder cancer (11). Dyrskjøt et al (12) bladder and Lee et al (13) bladder were 
2 independent studies with bladder cancer samples recorded in the Oncomine database. The information between G6PD expression and clinical significance were downloaded from The Cancer Genome Atlas (TCGA) database (https://cancergenome.nih.gov/) using the following searching terms: Project, TCGA-Bladder Urothelial Carcinoma; Primary site, bladder; expiration date, January 2018.

Cell culture. Human bladder cancer cell lines 5637 (thought to have the same molecular features as high-risk superficial bladder cancer) (14), T24 (thought to have the same molecular features as muscle invasive bladder cancer with grade III pathological grading) (15), TCCSUP (poorly differentiated and high-risk muscle invasive bladder cancer with grade IV pathological grading) (14), normal uroepithelial cell line SV-HUC-1 (16) and the engineered 293T cell line were purchased from the Cell Bank of the Chinese Academy of Sciences (Shanghai, China), and short tandem repeat DNA profiling analysis were performed by the supplier to validate all cell lines. 5637, T24 and TCCSUP cells were cultured in RPMI-1640 medium, SV-HUC-1 cells were cultured in Minimum Essential medium and 293T cells were cultured in DMEM. All the aforementioned mediums without any antibiotics (Gibco; Thermo Fisher Scientific, Inc., Waltham, MA, USA) were pre-supplemented with $10 \%$ fetal bovine serum (Gibco; Thermo Fisher Scientific, Inc.), and all cell lines were incubated under standard conditions $\left(37^{\circ} \mathrm{C}\right.$ and $5 \% \mathrm{CO}_{2}$ ).

Plasmid construction, lentiviral packaging and transfection. The G6PD-overexpression plasmid was acquired from Vigene Biosciences, Inc. (Rockville, MD, USA). Validated sequences of short hairpin RNA (shRNA) against G6PD (shG6PD) were screened from Sigma-Aldrich online (Sigma-Aldrich; Merck KGaA, Darmstadt, Germany). Scramble sequences were designed with the corresponding shG6PD sequences using the siRNA Wizard v3.1 online software (https://www.invivogen. com/sirnawizard/) declaring the absence of preclusive mRNA and miRNA seed sequence matches. Oligonucleotides of G6PD shRNA and control shRNA were then synthesized (Tsingke, Hangzhou, China) and inserted into the GIPZ lentiviral vector. The sequences used were as follows: shG6PD forward, 5'-CCGGCAACAGATACAAGAACGTGAACTCG AGTTCACGTTCTTGTATCTGTTGTTTTTG-3' and reverse, 5'-AATTCAAAAACAACAGATACAAGAACGTGAACTC GA GTTCACGTTCTTGTATCTGTTG-3'; Scramble forward, 5'-CCGGGCAAAGCAAACGTGACATAAACTCGAGTTTA TGTCACGTTTGCTTTGCTTTTTG-3' and reverse, 5'-AAT TCAAAAAGCAAAGCAAACGTGACATAAACTCGAGTT ATGTCACGTTTGCTTTGC-3'. Then the vectors were co-transfected with pSPAX2 and pMD2G (purchased from Addgene, Inc., Cambridge, MA, USA) plasmids (4:3:1 for vectors, pSPAX2 and pMD2G, respectively) into the $293 \mathrm{~T}$ cell line cultured in $60 \mathrm{~mm}$ plates (at $60 \%$ cell density) using a calcium phosphate precipitation method (17). The supernatant, which contained lentivirus, was harvested 48 or $72 \mathrm{~h}$ posttransfection. Subsequent to virus packaging, T24 and TCCSUP cells cultured in $60 \mathrm{~mm}$ plates (at $30 \%$ cell density) were infected for 48 or $72 \mathrm{~h}$ with polybrene $(5 \mu \mathrm{l} / \mathrm{ml}$; Sigma-Aldrich; Merck KGaA) and the medium was changed 6-12 h later.
Successfully transfected G6PD-shRNA cell lines were screened with $0.5 \mathrm{mg} / \mathrm{ml}$ puromycin (Sigma-Aldrich; Merck $\mathrm{KGaA}$ ) and the transfection efficiency was validated using green fluorescent protein $(488 \mathrm{~nm})$ expression and western blotting.

Cell counting kit-8(CCK-8) assay and colony formation assay. The proliferation of different groups of cells were compared using a CCK-8 kit (Dojindo Molecular Technologies, Inc., Kumamoto Japan) according to the manufacturer's protocol. 5637, T24 and TCCSUP cell lines with or without transfection were firstly prepared at a density of 1,000 cells/plate in 96-well plates under standard conditions $\left(37^{\circ} \mathrm{C}\right.$ and $\left.5 \% \mathrm{CO}_{2}\right)$. Then, premixed medium (as aforementioned) with a $10 \%$ concentration of CCK-8 reagent was added into each well and placed in standard conditions $\left(37^{\circ} \mathrm{C}\right.$ and $\left.5 \% \mathrm{CO}_{2}\right)$ for $1 \mathrm{~h}$ prior to measurement at an optical density of $450 \mathrm{~nm}$. The preparations of cells exposed to $10 \mu \mathrm{M}$ 6-aminonicotinamide (6-AN; Sigma-Aldrich; Merck KGaA), 0, 5, 10, 20, 40 or $80 \mu \mathrm{g} / \mathrm{ml}$ cisplatin (Sigma-Aldrich; Merck KGaA), 5 or $10 \mu \mathrm{M}$ SC79 (MedChemExpress, Monmouth Junction, NJ, USA) or controlled DMSO for 24 or $48 \mathrm{~h}$ following the same procedure. As for colony formation assay, cells were seeded at a density of 500 cells/plate in 6-well plates and cultured for 8-10 days under standard conditions followed by $15 \mathrm{~min}$ fixation at room temperature and 15 min staining $(0.5 \%$ crystal violet) at room temperature of the colonies which were performed prior to comparison.

Reverse transcription-quantitative polymerase chain reaction (RT-qPCR). TRIzol (Invitrogen; Thermo Fisher Scientific, Inc.) was used for cells total RNA extraction according to manufacturer's protocol. Takara PrimeScript ${ }^{\mathrm{TM}}$ RT and SYBR EX Taq ${ }^{\mathrm{TM}}$ kits (Takara Bio, Inc., Otsu, Japan) were used according to manufacturer's protocol. The specify thermocycling conditions used were as follows: Step 1, $95.0^{\circ} \mathrm{C}$ for $30 \mathrm{sec}$; step 2,40 cycles of $95^{\circ} \mathrm{C}$ for $5 \mathrm{sec}$ and $60^{\circ} \mathrm{C}$ for $30 \mathrm{sec}$; and step 3 , melt curve analysis at $65^{\circ} \mathrm{C}$ to $95^{\circ} \mathrm{C}$, increasing in $0.5^{\circ} \mathrm{C}$ increments for $5 \mathrm{sec}$. The primers were designed as follows: G6PD forward, 5'-ACCGCATCGACC ACTACCT-3' and reverse 5'-TGGGGCCGAAGATCCT GTT-3'; $\beta$-actin forward, 5'-GCAAGCAGGAGTATGAC GAG-3' and reverse, 5'-CAAATAAAGCCATGCCAATC-3'. Control groups were used to confirm the absence of the pollution of agents or primer dimers, and melt-curve analysis was used to identify the specificity of amplification. All genes were normalized to $\beta$-actin expression, and the gene mRNA relative expressions were calculated using the $\Delta \Delta \mathrm{Cq}$ method (18) using SPSS version 22.0 software (IBM Corp., Armonk, NY, USA).

Western blotting. 5637, T24 and TCCSUP cell lines were firstly washed using PBS twice prior to being lysed using RIPA Lysis buffer with $1 \%$ cocktail protease inhibitor (Thermo Fisher Scientific, Inc.) for $4 \mathrm{~h}$ at $4^{\circ} \mathrm{C}$ and purified by centrifugation $\left(4^{\circ} \mathrm{C}, 15,000 \times \mathrm{g}, 15 \mathrm{~min}\right)$. Subsequent to concentration measurement using a BCA protein assay (Pierce; Thermo Fisher Scientific, Inc.), $15 \mu \mathrm{g} / 10 \mu \mathrm{l}$ protein samples were loaded in $12 \%$ Tris-acetate gels (Invitrogen; Thermo Fisher Scientific, Inc.) and then separated by 
electrophoresis. Next, the proteins were transferred onto a polyvinylidene fluoride membrane. Then, the membrane was blocked with 5\% non-fat milk in Tris-buffered saline containing 1\% Tween-20 (TBST) for $1 \mathrm{~h}$ at room temperature and further incubated with primary antibodies for $12 \mathrm{~h}$ at $4^{\circ} \mathrm{C}$. Subsequent to washing with TBST three times, the membrane was incubated with secondary antibodies for $1 \mathrm{~h}$ at room temperature. The primary antibodies used in this experiment were: Rabbit polyclonal antibody G6PD (1:10,000; cat no. ab993; Abcam, Cambridge, UK); Rabbit monoclonal antibodies phosphorylated-protein kinase B (AKT; Ser473; 1:1,000; cat no. 4060; CST Biological Reagents Co., Ltd., Shanghai, China), AKT (1:1,000; cat no. 4685; CST Biological Reagents Co., Ltd.), P21 (1:2,000; cat no. ab109520; Abcam), P27 (1:2,000; cat no. ab32034; Abcam), cleaved caspase-3 (1:1,000; cat no. 9664; CST Biological Reagents Co., Ltd.), cleaved caspase-7 (1:1,000; cat no. 8438; CST Biological Reagents Co., Ltd.), cleaved caspase-9 (1:1,000; cat no. 7237; CST Biological Reagents Co., Ltd.) and Mouse monoclonal antibody $\beta$-actin (1:2,000; cat no. ab6276; Abcam). The secondary antibodies used in this experiment were: Goat anti Rabbit-horseradish peroxidase (HRP; 1:5,000; cat no. PDR007; Fdbio Science, Hangzhou, China) and Goat anti Mouse-HRP (1:5,000; cat no. PDM007; Fdbio Science). Immunodetection was performed by EZ-ECL chemiluminescence detection kit (Biological Industries, Kibbutz Beit Haemek, Israel). Protein bands were analyzed using Image-Pro Plus software 6.0 (Media Cybernetics, Inc., Rockville, MD, USA), and $\beta$-actin was selected as an internal reference.

Flow cytometry analysis of cell apoptosis. Prepared T24 and TCCSUP cell lines (60\% cell density in $6 \mathrm{~mm}$ dishes) with or without the shRNA-mediated G6PD knockdown were harvested with non-EDTA trypsin (Biological Industries), washed twice with cold PBS and then incubated using BD Annexin V-APC/7-aminoactinomycin D Apoptosis Detection Kit (BD Biosciences, San Jose CA, USA) according to the manufacturer's protocol. All aforementioned cells were harvested and stained with Annexin V (10 $\mu \mathrm{l} / 200 \mu \mathrm{l})$ and 7-aminoactinomycin D $(10 \mu \mathrm{l} / 200 \mu \mathrm{l})$ for $15 \mathrm{~min}$ in the dark at room temperature, and then apoptosis was analyzed using BD FACSCanto ${ }^{\mathrm{TM}}$ II (BD Biosciences), and data was then analyzed by FlowJo 7.6 software (FlowJo LLC, Ashland, OR, USA).

Intracellular reactive oxygen species (ROS) detection. T24, TCCSUP and 5637 cell lines with or without shRNA-mediated G6PD knockdown or G6PD overexpression or in the presence or absence of $\mathrm{H}_{2} \mathrm{O}_{2}$ were prepared in 9 wells in 96-well plates. Then, each group of cells was stimulated with or without $50 \mu \mathrm{M} \mathrm{H}_{2} \mathrm{O}_{2}$ for $30 \mathrm{~min}$ at $37^{\circ} \mathrm{C}$ prior to being co-incubated with $20 \mu \mathrm{M}$ diochloro-dihydro-fluorescein diacetate (Invitrogen; Thermo Fisher Scientific, Inc.) for 30-60 min at $37^{\circ} \mathrm{C}$. Then, 3 wells of each group of cells (selected as the cell counting group) were used for cell counting. The remaining wells (selected as the measuring group) were used to detect the ROS levels. Fluorescence was read at $485 \mathrm{~nm} / 520 \mathrm{~nm}$ by a fluorescent enzyme meter, Varioskan ${ }^{\mathrm{TM}}$ Flash (Thermo Fisher Scientific, Inc.). Levels of cellular ROS were normalized to the total number of cells.
Isobolographic analysis. In order to determine the combination effects of cisplatin and 6-AN, isobolographic analysis was performed. T24 and TCCSUP cell lines were firstly prepared at a density of 5,000 cells/plate in 96-well plates under standard conditions $\left(37^{\circ} \mathrm{C}\right.$ and $\left.5 \% \mathrm{CO}_{2}\right)$. Then cells were incubated with combination of different concentrations of 6-AN $(\mathrm{C} 1=0$, $0.5,1,4,8$ or $16 \mu \mathrm{M})$ and cisplatin $(\mathrm{C} 2=0,5,10,20,40$ or $80 \mu \mathrm{g} / \mathrm{ml}$ ). A total of $24 \mathrm{~h}$ later, premixed medium with a $10 \%$ concentration of CCK-8 reagent was added into each well and placed in standard conditions for $1 \mathrm{~h}$ prior to measurement at an optical density of $450 \mathrm{~nm}$. Once the background density was excluded, the $450 \mathrm{~nm}$ density (D) of each combination was used for cytotoxicity calculation using the following formula: Cytotoxic effect $(\mathrm{CE})=1-\mathrm{D}(\mathrm{C} 1, \mathrm{C} 2) / \mathrm{D}(0,0)$, and $\mathrm{D}(0,0)$ was selected as the $0 \%$ cytotoxic effect. Isoboles are defined as isoeffect curves that reveal the concentration of the combination of two drugs which results in a similar CE to a previous study $(19,20)$. The curves were obtained by ligaturing plots which represent the concentration combination of two drugs resulting in a $50 \% \mathrm{CE}$. The straight lines refer to the theoretical additivity line resulting in a $50 \% \mathrm{CE}$, and all data were analyzed using SPSS 22.0 (IBM Corp., Armonk, NY, USA) software.

Statistical analysis. SPSS 22.0 (IBM Corp.) was used to analyze the data. The normality of the data was initially determined using a Kolmogorov-Smirnov test. Data was presented as the mean \pm standard deviation. The correlations between G6PD expression and clinicopathological characteristics were analyzed using a Pearson's $\chi^{2}$ test or a continuity correction $\chi^{2}$ test. Overall survival and disease-free survival rates curves were plotted using the Kaplan-Meier method, and data was analyzed by a log-rank test. One-way analysis of variance test was used to examine the differences between different groups. Student-Newman-Keuls test was used as a post hoc test. $\mathrm{P}<0.05$ was considered to indicate a statistically significant difference. Data was derived from at least 3 repeated experiments.

\section{Results}

High G6PD expression is a poor prognostic factor in bladder cancer. To investigate whether G6PD expression is associated with bladder cancer progression and prognosis, the present study compared the G6PD expression levels in bladder cancer using the Oncomine database. G6PD mRNA expression levels were significantly higher in bladder cancer tissues compared with that in adjacent normal tissues $(\mathrm{P}<0.05$; Fig. 1A and $\mathrm{B})$. Furthermore, the levels of G6PD expression notably increased with increasing $\mathrm{T}$ stage (Fig. 1A and $\mathrm{B}$ ). In addition, the results of qPCR analysis and western blotting revealed that G6PD mRNA levels were significantly upregulated in three tumor cell lines compared with a normal urothelial cell line $(\mathrm{P}<0.05)$ and that the protein levels were notably upregulated. Furthermore, T24 and TCCSUP, which have a higher malignant potential compared with 5637, exhibited higher G6PD expression levels compared with 5637 (Fig. 1C and D). Next, the clinical significance of G6PD in 408 patients with muscleinvasive bladder cancer (MIBC) from the TCGA database was investigated. Analysis revealed a significant association 


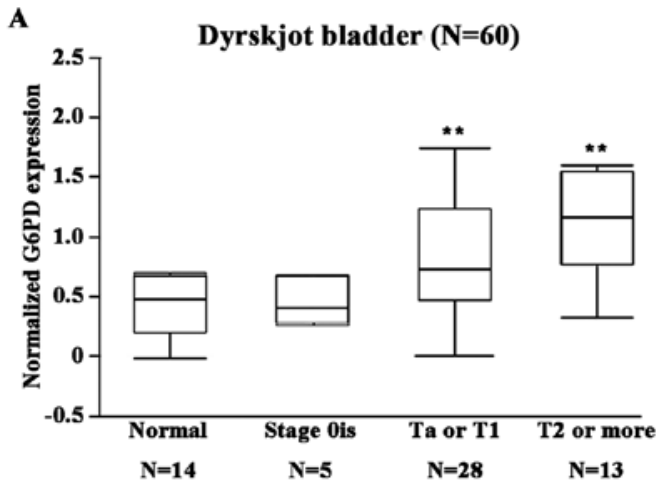

B

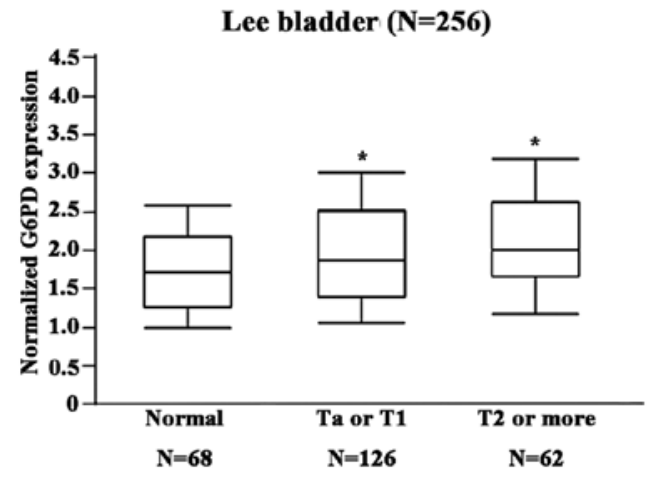

C
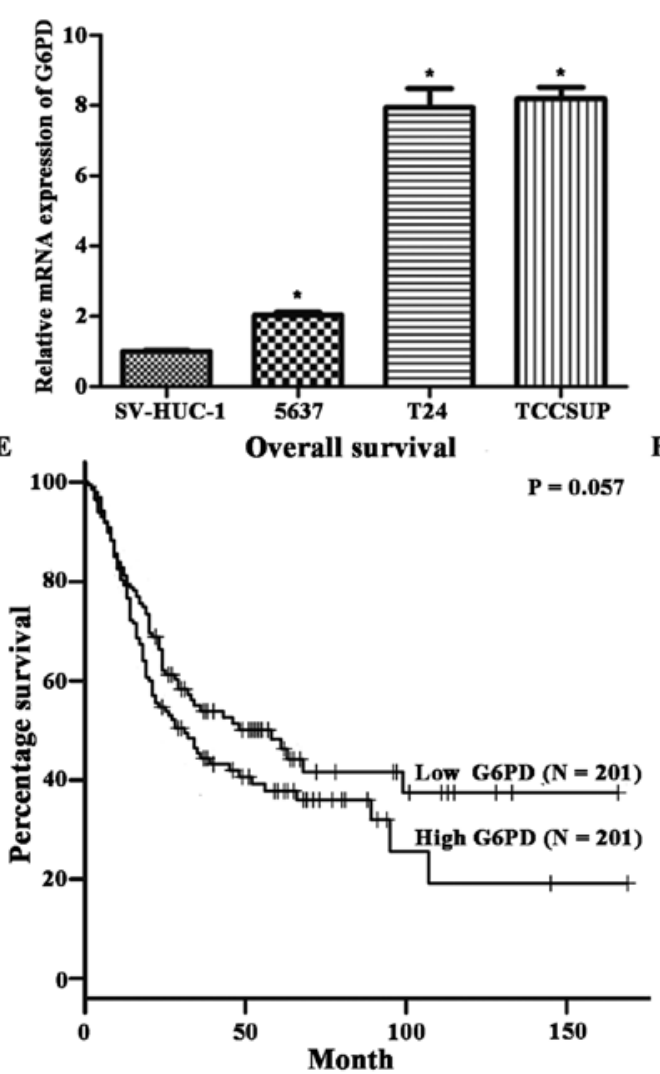

D SV-HUC-1 $5637 \quad$ T24 TCCSUP
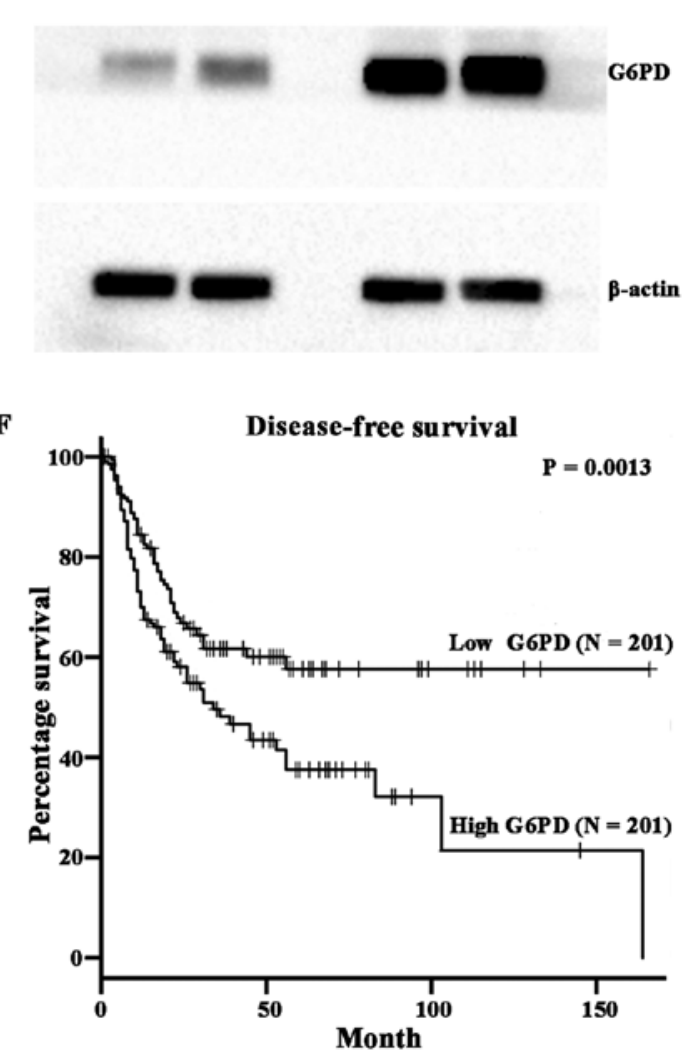

Figure 1. High G6PD expression is a poor prognostic factor in bladder cancer. (A) G6PD mRNA expression in bladder cancer and normal tissues from the Oncomine database $(\mathrm{N}=60) .{ }^{* *} \mathrm{P}<0.01$ Ta or $\mathrm{T} 1 \mathrm{vs}$. normal and ${ }^{* *} \mathrm{P}<0.01 \mathrm{~T} 2$ or more vs. normal. (B) G6PD mRNA expression in bladder cancer and normal tissues from the Oncomine database ( $\mathrm{N}=256$ ). ${ }^{*} \mathrm{P}<0.05$ Ta or $\mathrm{T} 1$ vs. normal and ${ }^{*} \mathrm{P}<0.05 \mathrm{~T} 2$ or more vs. normal. (C) G6PD mRNA expression levels in bladder cancer cell lines (5637, T24 and TCCSUP) and in normal human uroepithelial cells (SV-HUC-1). ${ }^{*} \mathrm{P}<0.055637$ vs. SV-HUC-1, ${ }^{\mathrm{P}}<0.05$ T24 vs. SV-HUC-1 and ${ }^{*} \mathrm{P}<0.05$ TCCSUP vs. SV-HUC-1. (D) G6PD protein expression in bladder cancer cell lines (5637, T24 and TCCSUP) and in normal human uroepithelial cells (SV-HUC-1). Kaplan-Meier analysis of (E) overall survival rates and (F) disease-free survival rates stratified by low G6PD expression (N=201) and high G6PD expression from The Cancer Genome Atlas database ( $\mathrm{N}=201)$. The median was used as the dividing line, and G6PD upregulation was significantly negatively associated with overall and disease-free survival. rates G6PD, glucose-6-phosphate dehydrogenase.

of G6PD expression with stage $(\mathrm{P}<0.05)$ and sex $(\mathrm{P}<0.001)$ (Table I). Kaplan-Meier survival analysis of 402 patients with MIBC (as the data for 6 patients was inaccessible) revealed that patients with high G6PD expression levels had a worse overall survival rates $(\mathrm{P}=0.057$, close to 0.05$)$ and a significantly worse disease-free survival rates $(\mathrm{P}=0.0013)$ compared with those with lower G6PD expression levels (Fig. 1E and F).

Knockdown of G6PD suppresses cell proliferation and growth, while increasing intracellular ROS levels. In order to determine the effect of upregulated G6PD expression in bladder cancer cell lines highly expressing G6PD, T24 and TCCSUP cells were transfected with sh-G6PD lentivirus. The infection efficiency was validated by western blotting and fluorescence microscopy, and G6PD was demonstrated to be significantly lower in transfected cell lines compared with their respective scramble controls $(\mathrm{P}<0.05 ;$ Fig. $2 \mathrm{~A})$. The CCK8 cell proliferation assay revealed that the knockdown of G6PD in the two cell lines significantly reduced cell proliferation ( $\mathrm{P}<0.05$; Fig. $2 \mathrm{~B})$. Furthermore, the colony-forming ability of the two cell lines was substantially suppressed in the G6PD knockdown groups compared with the control 
Table I. Association between G6PD expression level and clinicopathological features of 408 patients with muscle-invasive bladder cancer in The Cancer Genome Atlas database.

\begin{tabular}{lccc}
\hline & \multicolumn{2}{c}{ G6PD expression levels } & \\
\cline { 2 - 3 } Characteristic & Low & High & P-value \\
\hline Sex & & & $<0.001^{\mathrm{b}}$ \\
Male & 104 & 151 & \\
Female & 100 & 53 & \\
Age (years) & & & 0.265 \\
$<65$ & 75 & 86 & \\
$\geq 65$ & 129 & 118 & \\
Tumor grade & & & \\
0.207 & & & \\
Low & 15 & 9 & \\
High & 189 & 195 & \\
Stage & & & \\
I or II & 76 & 56 & \\
III or IV & 128 & 148 & \\
Lymph node metastasis & & & \\
Absent & 148 & 56 & \\
Present & 131 & 73 & \\
Distant metastasis & & & \\
Absent & 201 & 3 & \\
Present & 196 & 8 & \\
\hline & & & \\
\hline
\end{tabular}

${ }^{\mathrm{a}} \mathrm{P}<0.05$; ${ }^{\mathrm{b}} \mathrm{P}<0.01$; ${ }^{\mathrm{c}}$ continuity correction. G6PD, glucose-6-phosphate dehydrogenase.

groups (Fig. 2C). No significant difference was observed between G6PD-overexpressing and control T24 or TCCSUP cell lines, however the overexpression of G6PD was observed in the 5637 cell line, which exhibited lower G6PD expression prior to transfection, in addition to enhanced cell proliferation and colony-forming abilities compared with the control group (Fig. 2B and C). Additionally, the knockdown of G6PD in the two cell lines resulted in significantly higher ROS accumulation compared with the corresponding control groups, which indicated a weaker ability to survive oxidative stress $(\mathrm{P}<0.05$; Fig. 2D) $(12,13)$.

Knockdown of G6PD induces intracellular apoptosis and suppresses the phosphorylated-AKT/AKT pathway. Multiple studies have reported that toxic ROS levels tend to induce apoptosis or other adverse reactions in cells (21-23). The present study compared the apoptosis between G6PD-knockdown and control groups. Interestingly, significantly increased apoptosis was demonstrated by flow cytometry analysis in the G6PD-knockdown groups compared with the control $(\mathrm{P}<0.05$; Fig. 2E), in addition to the significant upregulation of cleaved caspase-3, -7 and -9 levels in G6PD-knockdown cells compared with scramble control cells $(\mathrm{P}<0.05$; Fig. $3 \mathrm{~A})$. As reported in previous studies, the AKT pathway, which serves a vital function in the proliferation and apoptosis of tumor cells, was also revealed to sensitize cells to oxidative apoptosis $(24,25)$. In addition, the AKT signaling pathway was also reported to promote the progression of bladder cancer (26). Therefore the present study investigated whether the knockdown of G6PD additionally suppressed AKT signaling. The western blotting results revealed a significant decrease of phosphorylated AKT $(\mathrm{P}<0.05)$, but no significant change was observed of the total AKT in two G6PD-knockdown cell lines (T24 and TCCSUP) compared with the corresponding controls (Fig. 3B). Furthermore, P27, a cell cycle regulator known to be inhibited by AKT, was also revealed to be upregulated in G6PD-knockdown cell lines (Fig. 3B). Interestingly, a rescue assay with SC79, a specific AKT activator, successfully restored the partial effects of G6PD knockdown $(\mathrm{P}<0.05$; Fig. 4A). All this evidence suggests that the suppression of AKT signaling in G6PD-knockdown bladder cancer cells may exert a substantial tumor inhibitory effect.

Inhibition of G6PD activity with 6-AN exerts antineoplastic effects and functions synergistically with cisplatin. Subsequently, the effect of G6PD inhibition on T24 and TCCSUP cell lines using 6-AN, a competitive G6PD inhibitor, was examined. The specific effect of 6-AN on G6PD was confirmed by the fact that 6 -AN treatment in the two cell lines resulted in similar results with G6PD knockdown (Figs. 4B-D and 5A). The 6-AN treatment in the two cell lines significantly reduced cell proliferation determined using a CCK8 cell proliferation assay $(\mathrm{P}<0.05$; Fig. 4B) and resulted in a significantly higher ROS accumulation compared with the corresponding control groups ( $\mathrm{P}<0.05$; Fig. 2C). A rescue assay with SC79, a specific AKT activator, successfully restored the partial effects of the treatment of 6-AN $(\mathrm{P}<0.05$; Fig. 4D). Additionally, the significant upregulation of cleaved caspase-3, -7 and -9 levels in cells with 6-AN treatment compared with cells with DMSO treatment was observed $(\mathrm{P}<0.05$; Fig. 3A). Of note, it was revealed that the $\mathrm{T} 24$ and TCCSUP cell lines (high G6PD expression) displayed a higher sensitivity to 6-AN compared with the SVHUC and 5637 cell lines (lower G6PD expression), suggesting the specificity and rationale of targeting higher G6PD activity in bladder cancer $\left({ }^{*} \mathrm{P}<0.05\right.$; Fig. 5B). Interestingly, it was revealed that the level of G6PD protein increased subsequent to 6-AN treatment. 6-AN may be metabolized to 6-amino-NAD $(\mathrm{P}+)$, a competitive inhibitor of NAD(P+)-requiring processes, particularly G6PD. Additionally, it does not directly affect the expression of G6PD protein itself (27). Thus, the increase of G6PD may be explained as a compensatory increasing. Finally, the present study investigated whether 6-AN has the ability to enhance the antitumor effects of cisplatin, a classical drug mostly used in bladder cancer chemotherapy. In the T24 and TCCSUP cell lines, 6-AN and cisplatin functioned synergistically to enhance cytotoxicity in the CCK8 assay, and a substantial dose reduction with the combination of the two drugs by using isoeffective drug concentrations resulting in $50 \%$ of the cytotoxic effect was observed (Fig. 5C). All these cumulative results indicate that inhibition of G6PD activity by 6-AN may be a potential therapeutic method for bladder cancer, particularly in cases with high G6PD expression, and that the combination of cisplatin with 6-AN may optimize the clinical dose of cisplatin or minimize the cisplatin-associated side effects. 


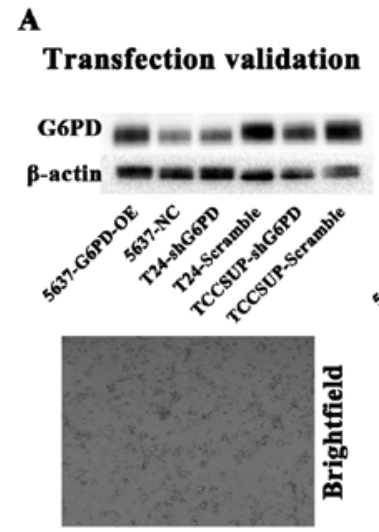

C

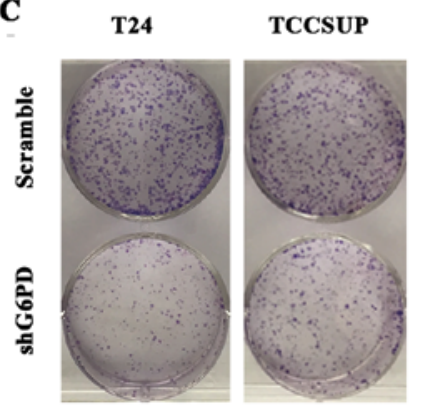

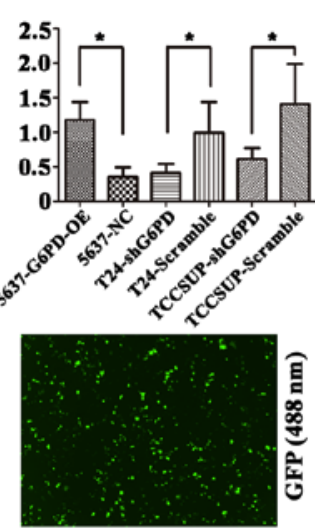

B

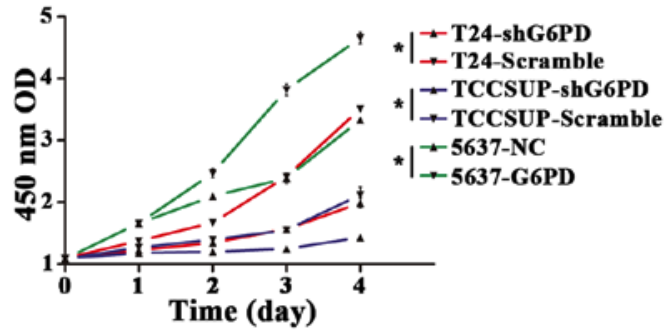

$\mathbf{D}$

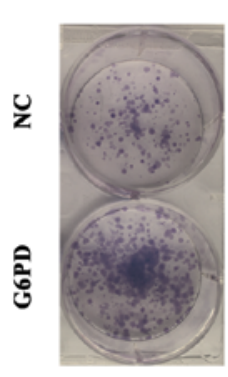

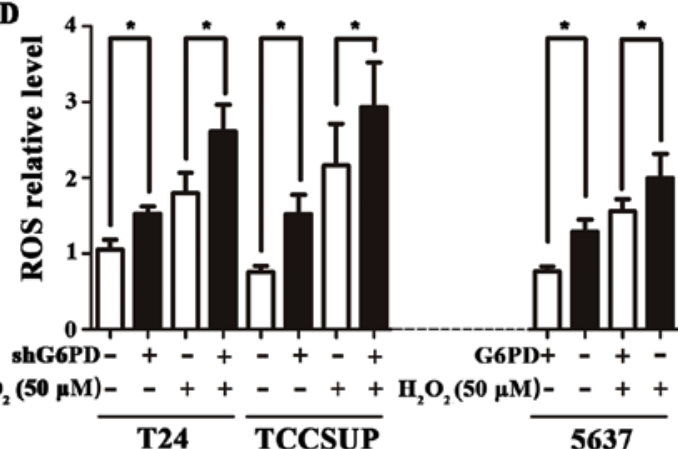

$\mathbf{E}$
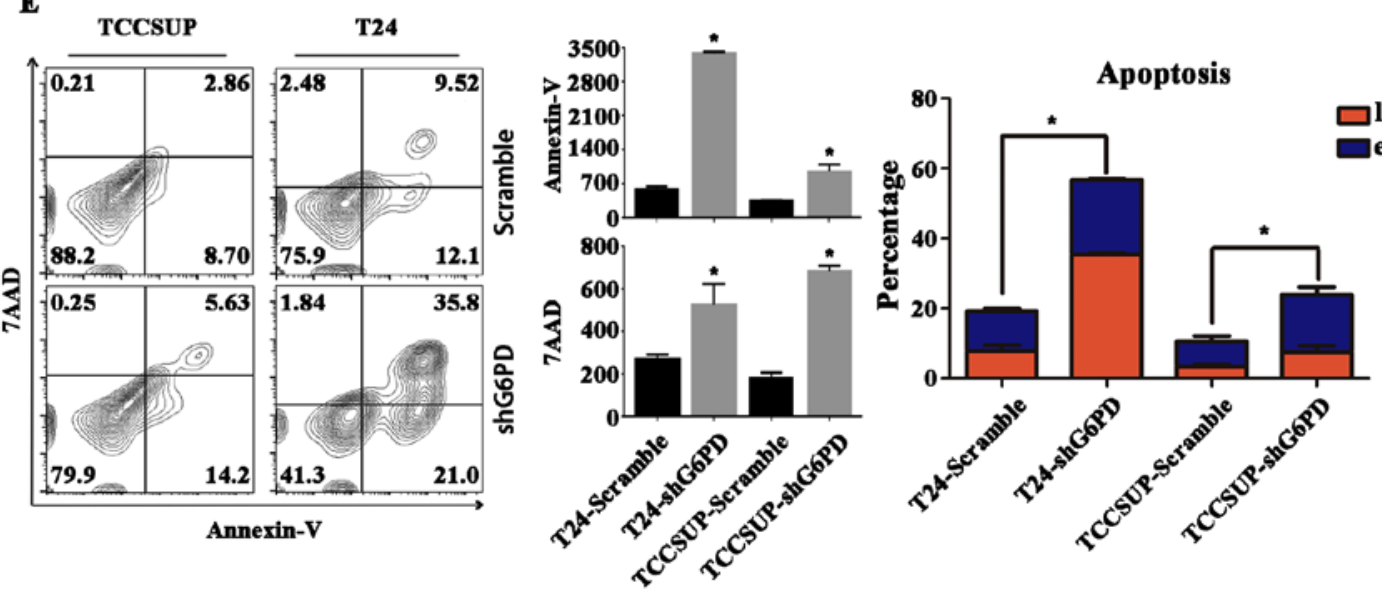

Figure 2. Knockdown of G6PD suppresses cell proliferation and growth while increasing intracellular ROS levels. (A) Transfection efficiency was validated by western blotting and fluorescence microscopy, and shG6PD reduced the expression of G6PD by at least $50 \%$. "P<0.05 with comparisons shown by lines. (B) Overexpression of G6PD promotes the proliferation of 5637 cell lines, whereas the inhibition of G6PD reduces the proliferation rate of T24 and TCCSUP cell lines as determined by a Cell Counting Kit- 8 assay. ${ }^{~} \mathrm{P}<0.05$ with comparisons shown by lines. (C) Colony formation assays of T24, TCCSUP and 5637 cell lines. (D) Relative ROS levels in T24, TCCSUP and 5637 cell lines with or without shRNA-mediated G6PD knockdown or G6PD overexpression or in the presence or absence of $\mathrm{H}_{2} \mathrm{O}_{2}(50 \mu \mathrm{g} / \mathrm{ml}),{ }^{*} \mathrm{P}<0.05$ with comparisons shown by lines. (E) Flow cytometric analysis presenting the percentage of apoptosis distribution between shG6PD-lentivirus- and scramble-lentivirus-transfected cells. Left, percentage of apoptosis in different groups of cell lines; Middle, enhancement of 2 types of apoptotic signals (Annexin V and 7AAD) in different groups of cell lines; Right, percentage of early and late apoptosis in different groups of cell lines. "P<0.05 with comparisons shown by lines. G6PD, glucose-6-phosphate dehydrogenase; ROS, reactive oxygen species; sh/shRNA, short hairpin RNA; 7AAD, 7-aminoactinomycin D; NC, negative control; OE, overexpression.

\section{Discussion}

It has become apparent that cancer cells require sugars to drive oncogenic processes $(28,29)$. For example, rapidly dividing cells require a constant supply of building blocks to maintain their elevated biosynthetic activity. In line with this increased metabolism are increased ROS levels (23). Considered to be by-products of oxygen consumption and cellular metabolism, ROS are formed by the partial reduction of molecular oxygen $(30,31)$. ROS homeostasis is crucial for cell survival and normal cell signaling, in addition to protecting cells from damage. To some extent, cancer cells must maintain cellular ROS at levels that favor growth (32). Glutathione (GSH), an important antioxidant for ROS detoxification, serves a key role in maintaining ROS homeostasis in cells (32). The content of activated GSH were revealed to be closely associated with the content of NADPH, the product of G6PD (23). G6PD is the rate-limiting enzyme in PPP; the oncogenic 
$\mathbf{A}$
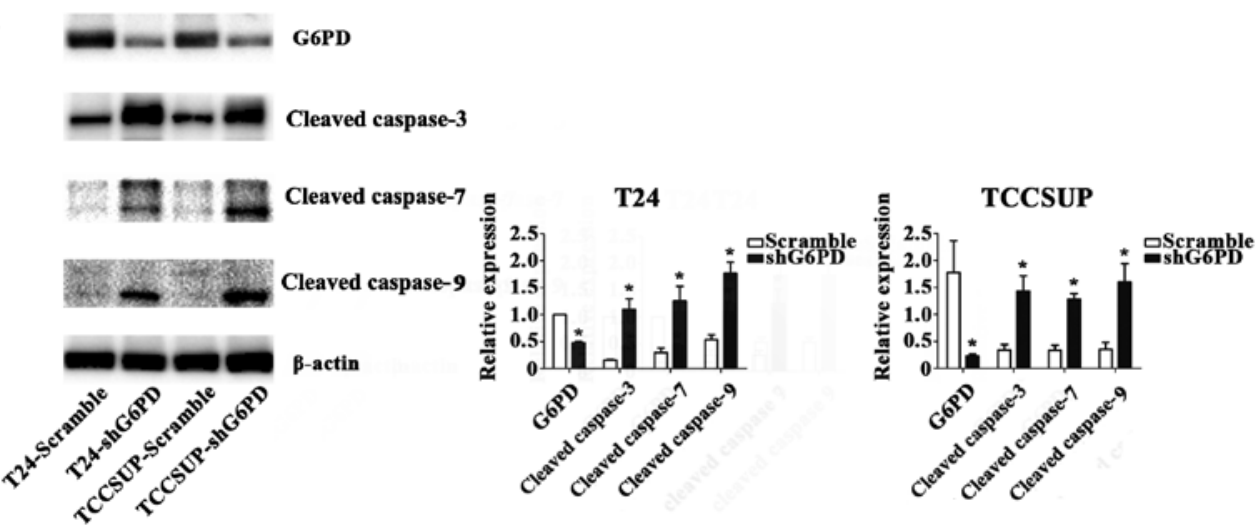

B
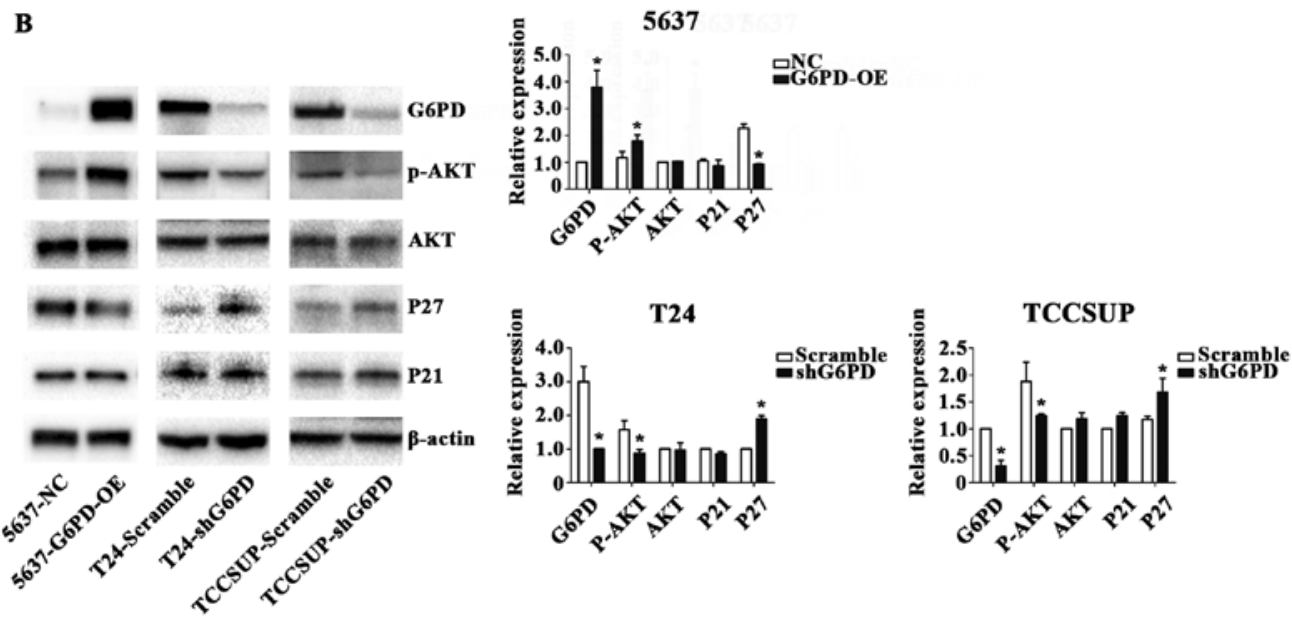

Figure 3. Knockdown of G6PD induced intracellular apoptosis and suppressed the p-AKT/AKT pathway. (A) Protein levels of G6PD, cleaved caspase-3, cleaved caspase-7, cleaved caspase-9 and $\beta$-actin are presented in two groups of cell lines. $\beta$-actin was used as a reference control. "P<0.05 vs. the scramble control. (B) Protein levels of G6PD, AKT, p-AKT, P21, P27 and $\beta$-actin are presented in three groups of cell lines. $\beta$-actin was used as a reference control. "P<0.05 vs. the scramble control. G6PD, glucose-6-phosphate dehydrogenase; p-, phosphorylated; AKT, protein kinase B; sh-, short hairpin RNA; NC, negative control; OE, overexpression.

$\mathbf{A}$

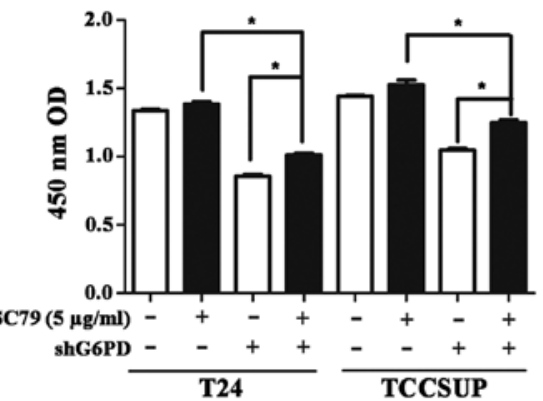

C

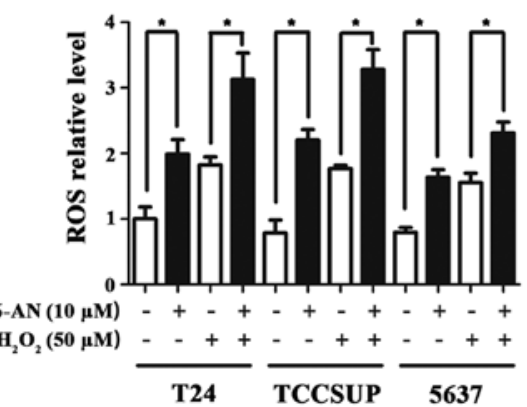

B

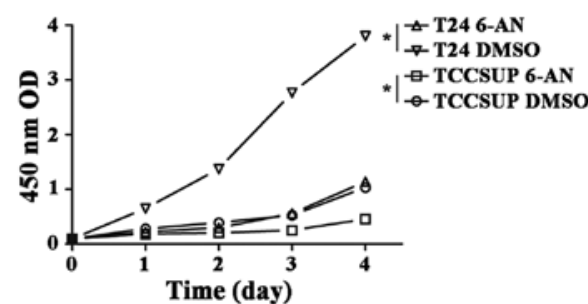

D

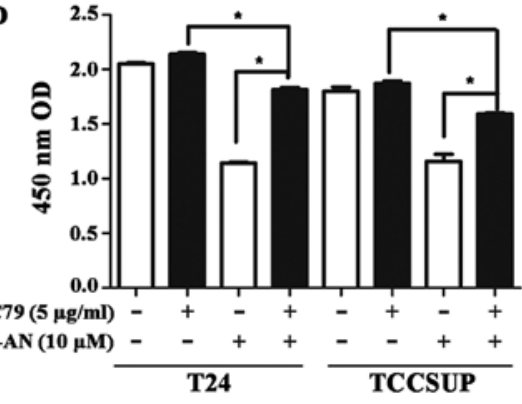

Figure 4. 6-AN treatment in two bladder cancer cell lines resulted in similar results to G6PD knockdown. (A) Rescue assay using $5 \mu \mathrm{g} / \mathrm{ml}$ SC79 for $24 \mathrm{~h}$ partially restored the loss of activity due to the shRNA-mediated knockdown of G6PD. * $\mathrm{P}<0.05$ with comparisons shown by lines. (B) Proliferation rate of T24 and TCCSUP cells was reduced by $6-\mathrm{AN}(5 \mu \mathrm{M})$, as determined by a Cell Counting Kit- 8 assay. Cells were seeded at a density of 2,000 cells/plate in 96-well plates at the start of the assay. 6-AN treatments were refreshed every $48 \mathrm{~h}$. $\mathrm{P}<0.05$ with comparisons shown by lines. (C) Relative ROS levels in T24 and TCCSUP cells in the presence or absence of 6 -AN $(10 \mu \mathrm{M})$ or $\mathrm{H}_{2} \mathrm{O}_{2}(50 \mu \mathrm{g} / \mathrm{ml}) .{ }^{*} \mathrm{P}<0.05$ with comparisons shown by lines. (D) Rescue assay using $5 \mu \mathrm{g} / \mathrm{ml}$ SC79 for $24 \mathrm{~h}$ partial restored the loss of activity due to 6 -AN $(10 \mu \mathrm{M})$-mediated inhibition of G6PD activity. * P<0.05 with comparisons shown by lines. OD, optical density; sh-, short hairpin RNA; G6PD, glucose-6-phosphate dehydrogenase; 6-AN, 6-aminonicotinamide; ROS, reactive oxygen species. 
$\mathbf{A}$

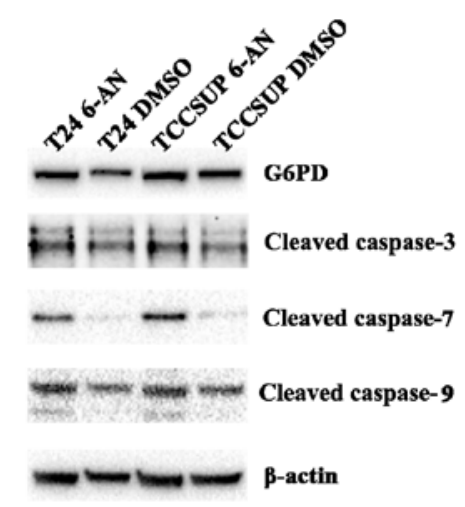

$\mathbf{C}$

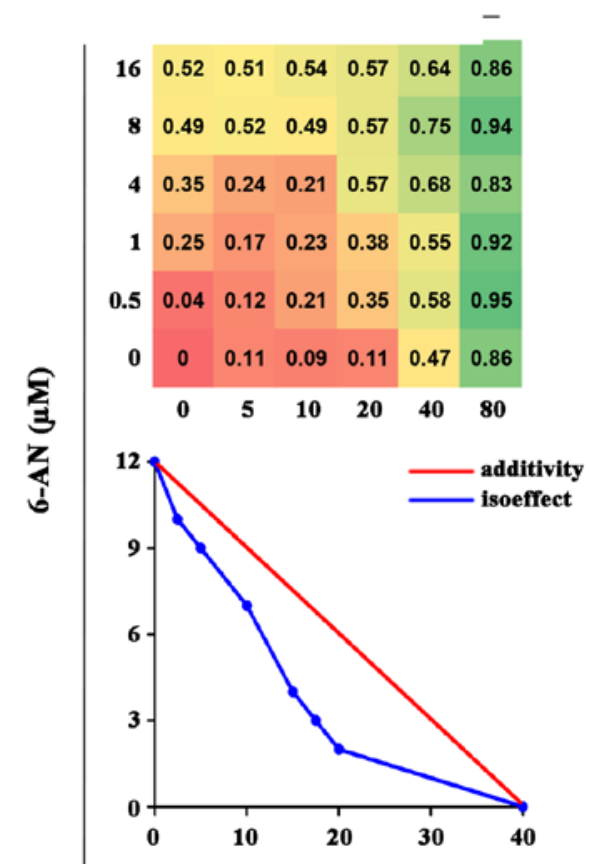

B

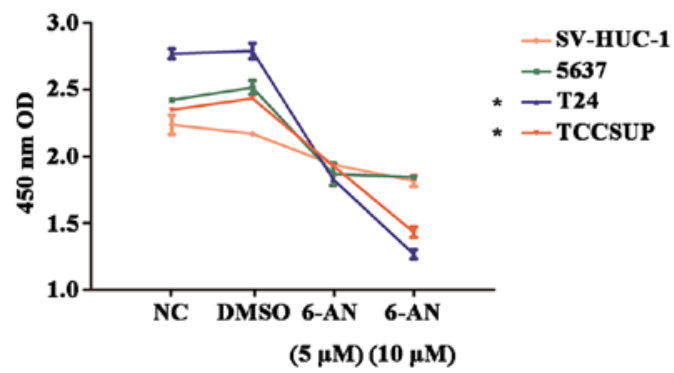

TCCSUP

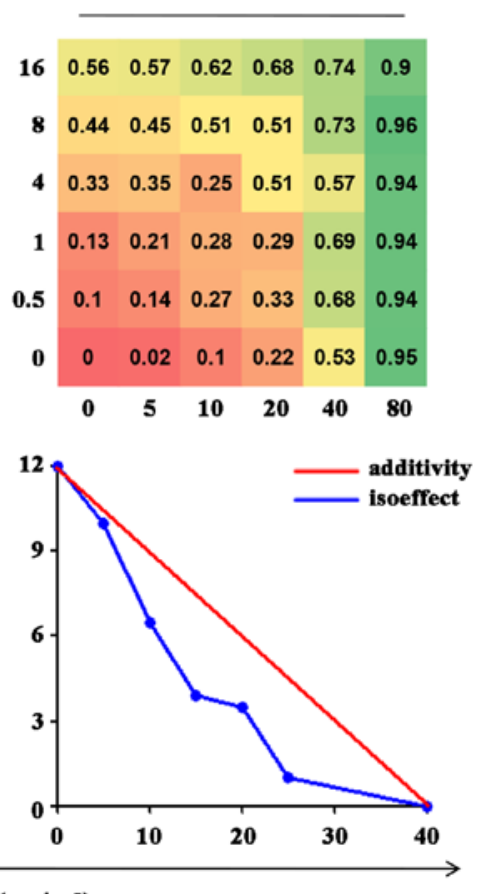

Cisplatin $(\mu \mathrm{g} / \mathrm{ml})$

Figure 5. 6-AN-mediated inhibition of G6PD activity induced antineoplastic effects and synergized with cisplatin. (A) Protein expression of G6PD, cleaved caspase-3, cleaved caspase-7, cleaved caspase- 9 and $\beta$-actin were presented in two groups of cell lines. $\beta$-actin was used as a reference control. (B) Cell proliferation was inhibited by different concentrations of 6 -AN $(5 \mu \mathrm{M}$ or $10 \mu \mathrm{M})$. All cell lines were seeded at a density of 1,000 cells/plate in 96 -well plates under standard conditions prior to comparison. ${ }^{*} \mathrm{P}<0.05$ vs. the SV-HUC-1. (C) Isobolographic analysis revealed that 6 -AN synergized with cisplatin. The curves were plotted using isoeffective drug concentrations that resulted in 50\% cytotoxicity. G6PD, glucose-6-phosphate dehydrogenase; 6-AN, 6-aminonicotinamide; NC, negative control; OD, optical density.

properties of G6PD have been attracting increasing attention and its increased activity in cancer cells was also recently demonstrated (33-35). In the present study, it was revealed that high G6PD expression was associated with a higher stage and poorer prognosis in patients with bladder cancer. G6PD expression was upregulated in tumor tissues compared with that in adjacent normal tissues, and the level of G6PD expression increased with increasing $\mathrm{T}$ stage. Analysis from the TCGA database revealed a significant association of G6PD expression with stage and sex in patients with MIBC. Patients with bladder cancer exhibited worse overall and disease-free survival rates compared with those with lower G6PD expression in the resected tumors.

Knockdown of G6PD in bladder cancer cell lines resulted in an increase of intracellular ROS levels. Inhibition of G6PD restricts the function of $\mathrm{PPP}$, resulting in lower NADPH production, an unstable GSH/oxidized GSH ratio and subsequent intracellular ROS accumulation, ultimately resulting in the disruption of the intracellular redox equilibrium (21-23). Consistent with the results of these previous studies, the toxic ROS levels induced apoptosis in bladder cancer cell lines, which may explain why the inhibition of G6PD suppressed the cell proliferation and colony formation ability of bladder cancer cell lines in vitro.

The AKT signaling pathway is known to serve a key role in the proliferation and apoptosis of tumor cells (24). An increasing number of studies demonstrated that activated AKT suppresses apoptosis by the phosphorylation of certain sites in $\mathrm{Bcl} 2$ associated agonist of cell death, caspase-9, protease-activated receptor 4 or other pro-apoptotic proteins $(36,37)$. AKT signaling was also reported to promote the progression of bladder cancer (25) and activated 
AKT signaling has been demonstrated to be positively correlated with tumor progression and poor clinical prognosis in patients with bladder cancer (38). The present results demonstrated that the knockdown of G6PD suppressed AKT signaling and increased the apoptosis of bladder cancer cells. This may explain the weakening of survival ability in G6PD-knockdown cancer cells. However, the detailed association between G6PD and the AKT pathway and the mechanism underlying the mode of action of AKT in bladder cancer requires further study.

6-AN, a competitive G6PD inhibitor (39), mostly used for radiosensitization combined with 2-deoxy-D-glucose (40-42), was also demonstrated to exert antineoplastic effects on cancer cells, alone or combined with other agents $(43,44)$. Similar to other studies, the present results demonstrated that G6PD inhibition with a fixed concentration of 6-AN significantly reduced the proliferation of bladder cancer cells, particularly those highly expressing G6PD, whereas it did not induce a substantial decrease of cell survival in normal cells $(\mathrm{P}<0.05$; Fig. 5B) (45). Furthermore, the results demonstrated that 6-AN functioned synergistically with cisplatin in bladder cancer treatment. Cisplatin-based chemotherapy remains the first-line chemotherapeutic treatment in patients with MIBC pre- and postoperatively (46). However, despite great success in tumor suppression, the application of cisplatin is restricted by drug resistance and side effects, the underlying mechanisms of which remain unclear (47). However, the hypothesis that the dysregulation of cell metabolism sustains drug resistance has gained the support of an increasing number of researchers $(9,10,48)$. Several agents are known to sensitize cancer cells to cisplatin, one of which is 6-AN (49). This effect is primarily due to the action of 6-amino-NAD $(\mathrm{P}+)$, which results in intracellular cisplatin enrichment and the accumulation of plasma tumor DNA adducts (50). Pretreatment with 6-AN has been demonstrated to sensitize different tumor cells to cisplatin cytotoxicity, even cisplatin-resistant cells; this may explain the synergistic effects of 6-AN and cisplatin on bladder cancer cells $(49,50)$. However, it has also been reported that 6-AN may cause neurotoxicity or hematological toxicity under certain conditions (43-46). Thus, further in vivo studies are required to optimize the therapeutic window and dose of 6 -AN in cancer treatment in clinical practice.

Altogether, the results of the present study demonstrate that high G6PD expression is associated with a higher stage and poorer prognosis in patients with bladder cancer. Inhibition of G6PD may suppress the growth of bladder cancer cells via ROS accumulation and AKT pathway suppression. Thus, targeting G6PD may be a potential therapeutic method for bladder cancer, particularly in cases with high G6PD expression. Furthermore, 6-AN used as a supplementary component in cisplatin-based chemotherapy may optimize the therapeutic doses of cisplatin, thereby minimizing the side effects (51).

\section{Acknowledgements}

The authors would like to thank The Key Laboratory of Combined Multi-Organ Transplantation, Ministry of Public Health (Hangzhou, China) for the use of the facilities and assistance from the technicians.

\section{Funding}

The present study was funded by the Key Project of the Science and Technology Program of Zhejiang Province (grant no. 2014C03028) and Science and Technology Program of Zhejiang Province (grant no. LY15H050002).

\section{Availability of data and materials}

The datasets used and/or analyzed during the current study are available from the corresponding author on reasonable request.

\section{Authors' contributions}

$\mathrm{XC}$ and $\mathrm{BJ}$ designed the experiments, performed the experiments, analyzed the data and wrote the paper. ZX, AC and GF performed the experiments. ZZ, YW and HP provided the reagents and helped with the experiments and the writing of the paper. All authors read and approved the final manuscript.

\section{Ethics approval and consent to participate}

Not applicable.

\section{Patient consent for publication}

Not applicable.

\section{Competing interests}

The authors declare that they have no competing interests.

\section{References}

1. Chen W, Zheng R, Baade PD, Zhang S, Zeng H, Bray F, Jemal A, $\mathrm{Yu}$ XQ and He J: Cancer statistics in China, 2015. CA Cancer J Clin 66: 115-132, 2016.

2. Bellmunt J, Orsola A, Leow JJ, Wiegel T, De Santis M, Horwich A and Group EGW; ESMO Guidelines Working Group: Bladder cancer: ESMO Practice Guidelines for diagnosis, treatment and follow-up. Ann Oncol 25 (Suppl 3): iii40-iii48, 2014.

3. Riganti C, Gazzano E, Polimeni M, Aldieri E and Ghigo D: The pentose phosphate pathway: An antioxidant defense and a crossroad in tumor cell fate. Free Radic Biol Med 53: 421-436, 2012.

4. Tian WN, Braunstein LD, Pang J, Stuhlmeier KM, Xi QC, Tian X and Stanton RC: Importance of glucose-6-phosphate dehydrogenase activity for cell growth. J Biol Chem 273: 10609-10617, 1998.

5. Hu T, Zhang C, Tang Q, Su Y, Li B, Chen L, Zhang Z, Cai T and Zhu Y: Variant G6PD levels promote tumor cell proliferation or apoptosis via the STAT3/5 pathway in the human melanoma xenograft mouse model. BMC Cancer 13: 251, 2013.

6. Batetta B, Pulisci D, Bonatesta RR, Sanna F, Piras S, Mulas MF Spano O, Putzolu M, Broccia G and Dessì S: G6PD activity and gene expression in leukemic cells from G6PD-deficient subjects. Cancer Lett 140: 53-58, 1999.

7. Van Driel BE, Valet GK, Lyon H, Hansen U, Song JY and Van Noorden CJ: Prognostic estimation of survival of colorectal cancer patients with the quantitative histochemical assay of G6PDH activity and the multiparameter classification program CLASSIF1. Cytometry 38: 176-183, 1999.

8. Polat MF, Taysi S, Gul M, Cikman O, Yilmaz I, Bakan E and Erdogan F: Oxidant/antioxidant status in blood of patients with malignant breast tumour and benign breast disease. Cell Biochem Funct 20: 327-331, 2002. 
9. Philipson KA, Elder MG and White JO: The effects of medroxyprogesterone acetate on enzyme activities in human endometrial carcinoma. J Steroid Biochem 23A: 1059-1064, 1985.

10. Zhang C, Zhang Z, Zhu Y and Qin S: Glucose-6-phosphate dehydrogenase: A biomarker and potential therapeutic target for cancer. Anticancer Agents Med Chem 14: 280-289, 2014.

11. Rhodes DR, Yu J, Shanker K, Deshpande N, Varambally R, Ghosh D, Barrette T, Pandey A and Chinnaiyan AM: ONCOMINE: A cancer microarray database and integrated data-mining platform. Neoplasia 6: 1-6, 2004.

12. Dyrskjøt L, Kruhøffer M, Thykjaer T, Marcussen N, JensenJL, Møller K and Ørntoft TF: Gene expression in the urinary bladder: A common carcinoma in situ gene expression signature exists disregarding histopathological classification. Cancer Res 64: 4040-4048, 2004.

13. Lee JS, Leem SH, Lee SY, Kim SC, Park ES, Kim SB, Kim SK, Kim YJ, Kim WJ and Chu IS: Expression signature of E2F1 and its associated genes predict superficial to invasive progression of bladder tumors. J Clin Oncol 28: 2660-2667, 2010.

14. Fogh J: Cultivation, characterization, and identification of human tumor cells with emphasis on kidney, testis, and bladder tumors. Natl Cancer Inst Monogr 49: 5-9, 1978.

15. Bubeník J, Baresová M, Viklický V, Jakoubková J, Sainerová H and Donner J: Established cell line of urinary bladder carcinoma (T24) containing tumour-specific antigen. Int J Cancer 11: 765-773, 1973.

16. Christian BJ, Loretz LJ, Oberley TD and Reznikoff CA: Characterization of human uroepithelial cells immortalized in vitro by simian virus 40. Cancer Res 47: 6066-6073, 1987.

17. Graham FL and van der Eb AJ: A new technique for the assay of infectivity of human adenovirus 5 DNA. Virology 52: 456-467, 1973.

18. Livak KJ and Schmittgen TD: Analysis of relative gene expression data using real-time quantitative PCR and the 2(-Delta Delta C(T)). Method 25: 402-408, 2001.

19. Loewe $\mathrm{S}$ : The problem of synergism and antagonism of combined drugs. Arzneimittelforschung 3: 285-290, 1953.

20. Tallarida RJ: An overview of drug combination analysis with isobolograms. J Pharmacol Exp Ther 319: 1-7, 2006.

21. Ichijo H, Nishida E, Irie K, ten Dijke P, Saitoh M, Moriguchi T, Takagi M, Matsumoto K, Miyazono K and Gotoh Y: Induction of apoptosis by ASK1, a mammalian MAPKKK that activates SAPK/JNK and p38 signaling pathways. Science 275: 90-94, 1997.

22. Moon DO, Kim MO, Choi YH, Hyun JW, Chang WY and Kim GY: Butein induces G(2)/M phase arrest and apoptosis in human hepatoma cancer cells through ROS generation. Cancer Lett 288: 204-213, 2010.

23. Moloney JN and Cotter TG: ROS signalling in the biology of cancer. Semin Cell Dev Biol 80: 50-64, 2018.

24. Vivanco I and Sawyers CL: The phosphatidylinositol 3-Kinase AKT pathway in human cancer. Nat Rev Cancer 2: 489-501, 2002.

25. Nogueira V, Park Y, Chen CC, Xu PZ, Chen ML, Tonic I, Unterman $\mathrm{T}$ and Hay $\mathrm{N}$ : Akt determines replicative senescence and oxidative or oncogenic premature senescence and sensitizes cells to oxidative apoptosis. Cancer Cell 14: 458-470, 2008.

26. Calderaro J, Rebouissou S, de Koning L, Masmoudi A, Hérault A, Dubois T, Maille P, Soyeux P, Sibony M, de la Taille A, et al: PI3K/AKT pathway activation in bladder carcinogenesis. Int J Cancer 134: 1776-1784, 2014.

27. Street JC, Alfieri AA and Koutcher JA: Quantitation of metabolic and radiobiological effects of 6-aminonicotinamide in RIF-1 tumor cells in vitro. Cancer Res 57: 3956-3962, 1997.

28. Ward PS and Thompson CB: Metabolic reprogramming: A cancer hallmark even warburg did not anticipate. Cancer Cell 21: 297-308, 2012

29. Wittig R and Coy JF: The role of glucose metabolism and glucose-associated signalling in cancer. Perspect Medicin Chem 1: $64-82,2008$

30. Giorgio M, Trinei M, Migliaccio E and Pelicci PG: Hydrogen peroxide: A metabolic by-product or a common mediator of ageing signals? Nat Rev Mol Cell Biol 8: 722-728, 2007.

31. Zorov DB, Juhaszova M and Sollott SJ: Mitochondrial reactive oxygen species (ROS) and ROS-induced ROS release. Physiol Rev 94: 909-950, 2014.
32. Vander Heiden MG, Cantley LC and Thompson CB: Understanding the Warburg effect: The metabolic requirements of cell proliferation. Science 324: 1029-1033, 2009.

33. Kuo W, Lin J and Tang TK: Human glucose-6-phosphate dehydrogenase (G6PD) gene transforms NIH 3T3 cells and induces tumors in nude mice. Int J Cancer 85: 857-864, 2000.

34. Jiang P, Du W and Yang X: A critical role of glucose-6-phosphate dehydrogenase in TAp73-mediated cell proliferation. Cell Cycle 12: 3720-3726, 2013

35. Rao X, Duan X, Mao W, Li X, Li Z, Li Q, Zheng Z, Xu H, Chen M, Wang PG, et al: O-GlcNAcylation of G6PD promotes the pentose phosphate pathway and tumor growth. Nat Commun 6: 8468, 2015.

36. Song G, Ouyang G and Bao S: The activation of Akt/PKB signaling pathway and cell survival. J Cell Mol Med 9: 59-71, 2005.

37. Goswami A, Burikhanov R, de Thonel A, Fujita N, Goswami M, Zhao Y, Eriksson JE, Tsuruo T and Rangnekar VM: Binding and phosphorylation of par- 4 by akt is essential for cancer cell survival. Mol Cell 20: 33-44, 2005.

38. Sun $\mathrm{CH}$, Chang YH and Pan CC: Activation of the PI3K/Akt/ mTOR pathway correlates with tumour progression and reduced survival in patients with urothelial carcinoma of the urinary bladder. Histopathology 58: 1054-1063, 2011.

39. Hothersall JS, Gordge M and Noronha-Dutra AA: Inhibition of NADPH supply by 6-aminonicotinamide: Effect on glutathione, nitric oxide and superoxide in J774 cells. FEBS Lett 434: 97-100, 1998.

40. Sharma PK and Varshney R: 2-Deoxy-D-glucose and 6-aminonicotinamide-mediated Nrf2 down regulation leads to radiosensitization of malignant cells via abrogation of GSH-mediated defense. Free Radic Res 46: 1446-1457, 2012.

41. Sharma PK, Bhardwaj R, Dwarakanath BS and Varshney R: Metabolic oxidative stress induced by a combination of 2-DG and 6-AN enhances radiation damage selectively in malignant cells via non-coordinated expression of antioxidant enzymes. Cancer Lett 295: 154-166, 2010.

42. Varshney R, Gupta S and Dwarakanath BS: Radiosensitization of murine Ehrlich ascites tumor by a combination of 2-deoxy-Dglucose and 6-aminonicotinamide. Technol Cancer Res Treat 3: 659-663, 2004.

43. Stolfi RL, Colofiore JR, Nord LD, Koutcher JA and Martin DS: Biochemical modulation of tumor cell energy: Regression of advanced spontaneous murine breast tumors with a 5-fluorouracil-containing drug combination. Cancer Res 52: 4074-4081, 1992.

44. Koutcher JA, Alfieri AA, Stolfi RL, Devitt ML, Colofiore JR, Nord LD and Martin DS: Potentiation of a three drug chemotherapy regimen by radiation. Cancer Res 53: 3518-3523, 1993.

45. Poulain L, Sujobert P, Zylbersztejn F, Barreau S, Stuani L, Lambert M, Palama TL, Chesnais V, Birsen R, Vergez F, et al: High mTORC1 activity drives glycolysis addiction and sensitivity to G6PD inhibition in acute myeloid leukemia cells. Leukemia 31: 2326-2335, 2017

46. Alfred Witjes J,Lebret T, Compérat EM, Cowan NC, De Santis M, Bruins HM, Hernández V, Espinós EL, Dunn J, Rouanne M, et al: Updated 2016 EAU Guidelines on muscle-invasive and metastatic Bladder Cancer. Eur Urol 71: 462-475, 2017.

47. Köberle B, Tomicic MT, Usanova S and Kaina B: Cisplatin resistance: Preclinical findings and clinical implications. Biochim Biophys Acta 1806: 172-182, 2010.

48. Liu H, Liu Y and Zhang JT: A new mechanism of drug resistance in breast cancer cells: Fatty acid synthase overexpression-mediated palmitate overproduction. Mol Cancer Ther 7: 263-270, 2008.

49. Budihardjo II, Walker DL, Svingen PA, Buckwalter CA, Desnoyers S, Eckdahl S, Shah GM, Poirier GG, Reid JM, Ames MM, et al: 6-Aminonicotinamide sensitizes human tumor cell lines to cisplatin. Clin Cancer Res 4: 117-130, 1998.

50. Catanzaro D, Gaude E, Orso G, Giordano C, Guzzo G, Rasola A, Ragazzi E, Caparrotta L, Frezza C and Montopoli M: Inhibition of glucose-6-phosphate dehydrogenase sensitizes cisplatinresistant cells to death. Oncotarget 6: 30102-30114, 2015.

51. Zhelev Z, Ivanova D, Bakalova R, Aoki I and Higashi T: Inhibition of the pentose-phosphate pathway selectively sensitizes leukemia lymphocytes to chemotherapeutics by ROS-independent mechanism. Anticancer Res 36: 6011-6020, 2016. 